\title{
Association among Parkinsonism-related motor complaints, cerebral small vessel disease, and cerebrovascular risk factors in a community- dwelling population
}

\section{Yang Guo}

Peking Union Medical College Hospital

Cai-hong Ji

Zhejiang University School of Medicine First Affiliated Hospital

Fei Han

Department of Neurology, Peking Union Medical College Hospital

Jiang-tao Zhang

Department of Neurology, Peking Union Medical College Hospital

Fei-fei Zhai

Department of Anesthesiology, Peking Union Medical College Hospital

\section{Li-xin Zhou}

Department of Neurology, Peking Union Medical College Hospital Jun Ni

Department of Neurology, Peking Union Medical College Hospital

Ming Yao

Department of Neurology, Peking Union Medical College Hospital

\section{Zheng-yu Jin}

Department of Radiology, Peking Union Medical College Hospital

\section{Shu-yang Zhang}

Department of Cardiology, Peking Union Medical College Hospital

\section{Li-ying Cui}

Department of Neurology, Peking Union Medical College Hospital

Yicheng Zhu ( $\nabla$ zhuych910@163.com )

\section{Research article}

Keywords: motor complaints, Parkinsonism, cerebral small vessel disease, cerebrovascular risk factors, hypertension, community-dwelling population

Posted Date: October 10th, 2019 
DOI: https://doi.org/10.21203/rs.2.15858/v1

License: (c) (1) This work is licensed under a Creative Commons Attribution 4.0 International License. Read Full License 


\section{Abstract}

Background Parkinsonism-related motor complaints are commonly seen in the elderly. Our study aimed to investigate the association among Parkinsonism-related motor complaints, cerebral small vessel disease and cerebrovascular risk factors in a community-dwelling population in a Chinese rural area.

Methods Individuals who were 50 years old or older, were independently living, were well-functioning, and had no history of ischemic or hemorrhagic stroke, were included. Brain magnetic resonance imaging (MRI), quantified motor function assessment, and questionnaire screening for Parkinsonism-related motor complaints were performed. Clinical data including cerebrovascular risk factors were collected. In univariate analysis, Chi-square test and student t-test were used to compare dichotomous variables and continuous variables, respectively, between individuals with or without motor complaints. In multivariate analysis, binary Logistic regression models were generated to determine risk factors for Parkinsonismrelated motor complaints. General linear models were used to compare motor parameters between individuals with or without motor complaints.

Results In the final analysis, 854 people were included. Individuals with motor complaints had a longer time for finger taping (6.2s v.s. $5.6 \mathrm{~s}, \mathrm{p}=0.006)$, and a longer time for $3 \mathrm{~m}$-walking( $4.0 \mathrm{~s}$ v.s. $3.6 \mathrm{~s}, \mathrm{p}=0.034$ ) than did those without motor complaints. Hypertension was associated with motor complaints (odds ratio, 1.82; $95 \%$ confidence interval [CI], [1.21, 2.73]; $p=0.004)$. Age was not associated with motor complaints; none of the neuroimaging markers of cerebral small vessel disease was associated with motor complaints.

Conclusion Hypertension is associated with Parkinsonism-related motor complaints. Better management of hypertension may prevent mobility limitation in the elderly. The questionnaire that we used for Parkinsonism is not suitable for screening small vessel disease in a community-dwelling population.

\section{Background}

Signs of Parkinsonism have been found to be increased with age and are often considered as benign concomitants of aging. Recent studies have shown that in a community-dwelling population, mild Parkinsonian syndrome[1-3] and gait impairment[4-7] are related to neuroimaging markers of cerebral small vessel disease (CSVD) such as white matter hyperintensity (WMH), lacunes, cerebral microbleeds (CMB), and brain atrophy. In addition, Parkinsonian signs are associated with mortality[8]. A crosssectional study showed that motor deficits of upper and lower extremities are associated with CSVD, especially with WMH and brain atrophy[9]. Moreover, hypertension, as a cerebrovascular risk factor, is associated with decreased gait speed in the elderly population[10].

These studies were all conducted by neurologists or medical staff trained to assess motor deficits, which were time- and resource-consuming. Because of the very high rate of undiagnosed Parkinsonism, a screening method based on a questionnaire for patients' motor complaints was built and was proven to be effective in enhancing recognition of incipient Parkinsonism[11-13]. Considering that both 
neuroimaging changes of CSVD and motor deficits are highly prevalent in the general aging population, we hypothesize that the questionnaire for Parkinsonism-related motor complaints might also be useful in screening for CSVD in the general population. Therefore, we investigated the association among Parkinsonism-related motor complaints quantified using a questionnaire, neuroimaging markers of CSVD, and cerebrovascular risk factors in a population-based study.

\section{Methods}

- Inclusion and exclusion criteria

Our study was based on the the Shunyi Study, a population-based prospective cohort study designed to investigate the risk factors and consequences of brain changes in community-dwelling adults in the Chinese population. Between June 2013 and April 2016, inhabitants from 5 villages of Shunyi, a suburb district of Beijing, who were 35 years or older and independently living, were invited to participate. Standard baseline assessments including structured questionnaires, physical examination, and laboratory tests were accomplished. We included participants accomplished MRI study and Tanner's standard questionnaire. Participants were excluded if they had a history of ischemic/hemorrhagic stroke or muscle strength less than 4 of any limb. Participants younger than 50 years old were also excluded since motor complaints were rarely seen in this group.

- Imaging parameters

MRI acquisition was performed from July 2014 to April 2016 using a single 3-Tesla Siemens Skyra scanner (Siemens, Erlangen, Germany). Three-dimensional T1-weighted images were acquired using magnetization-prepared rapid gradient-echo sequences in the sagittal plane [repetition time (TR) $=$ $2,530 \mathrm{~ms}$; echo time $(\mathrm{TE})=3.43 \mathrm{~ms}$; inversion time $=1,100 \mathrm{~ms}$; field of view $(\mathrm{FOV})=256 \times 256 \mathrm{~mm}$; voxel size $=1 \times 1 \times 1.3 \mathrm{~mm}$; flip angle $=8 ; 144$ sagittal slices $]$. T2-weighted images $(\mathrm{TR}=6,000 \mathrm{~ms} ; \mathrm{TE}=125 \mathrm{~ms}$; FOV $=230 \times 230 \mathrm{~mm}$; flip angle $=90$; slice thickness $=5 \mathrm{~mm} ;$ gap $=1 \mathrm{~mm} ; 80$ axial slices), fluid-attenuated inversion recovery (FLAIR) images $(T R=8,500 \mathrm{~ms} ; \mathrm{TE}=81 \mathrm{~ms} ; \mathrm{FOV}=230 \times 230 \mathrm{~mm}$; flip angle $=150$; slice thickness $=5 \mathrm{~mm}$; gap $=1 \mathrm{~mm} ; 80$ axial slices), and susceptibility weighted imaging (SWI) images $(\mathrm{TR}=$ $27 \mathrm{~ms} ; \mathrm{TE}=20 \mathrm{~ms} ; \mathrm{FOV}=208 \times 230 \mathrm{~mm}$; flip angle: 15 ; slice thickness $=1.5 \mathrm{~mm} ; 80$ axial slices) were acquired in the axial plane.

- Definition of cerebrovascular risk factors

Hypertension was defined as blood pressure $\geq 140 / 90 \mathrm{mmHg}$; diabetes mellitus was defined as a fasting plasma glucose level $\geq 7.0 \mathrm{mmol} / \mathrm{L}$ or 2 -h plasma glucose level $\geq 11.1 \mathrm{mmol} / \mathrm{L}$ during an oral glucose tolerance test; hyperlipidemia was defined as total cholesterol $>5.2 \mathrm{mmol} / \mathrm{L}$ or low-density lipoprotein > $2.58 \mathrm{mmol} / \mathrm{L}$; and smoking was defined as an individual smoking at least 1 cigarette per day for more than 6 months before enrollment.

- Definition of MRI imaging markers of CSVD 
The neuroimaging markers for CSVD, including WMH, lacunes, CMBs, and dilated perivascular spaces (PVS), were defined according to the STRIVE standard, 2013[14]. Periventricular WMH (PVWMH) and deep WMH (DWMH) were scored on axial FLAIR images using the Fazekas scale[15]. PVWMH and DWMH were considered mild if it scored 0 or 1 on the Fazekas scale, and severe if it scored more than 2 on the Fazekas scale. Total WMH was defined as mild if the PVWMH or DWMH was severe. The severity of dilated PVS was scored using a previously published method[16]. Dilated PVS was considered severe if it was degree 2 or more. Automated segmentation of 3D-T1 images was used to obtain the probability maps of gray matter (GM), white matter [10], and cerebral spinal fluid (CSF). Total brain volume was than calculated as the sum of the volumes of GM, WM, and CSF. The brain parenchymal fraction (BPF) was defined as the ratio of total brain volume to intracranial volume.

WMH, lacunes, CMBs, and PVS were independently rated by well-trained researchers who were blinded to the clinical data. Intra-rater agreement was assessed in a random sample of 50 individuals with an interval of at least 1 month between the first and second readings. Kappa values for the intra-rater agreements were 0.84 for $\mathrm{PVWMH}, 0.89$ for $\mathrm{DWMH}, 0.73$ for lacunes, 0.90 for $\mathrm{CMBs}, 0.71$ for $\mathrm{PVS}$ in the basal ganglia (PVS-BG), and 0.61 for PVS in WM (PVS-WM).

\section{- Assessment of motor function}

Participants in the Shunyi cohort were all invited to a motor function assessment of upper and lower extremities[9]. Times for 3-m walking, 5-repeat chair-stand, 10-repeat pronation-supination, and 10-repeat finger-tapping were used in our study.

- Statistical analysis

Continuous variables were expressed as mean and standardized deviation, and categorical variables were expressed as frequencies and proportions. All statistical analyses were performed using IBM-SPSS version 22.0 .

Individuals were assigned into the motor complaint group if they had a score 1 or more on the questionnaire. Otherwise they were assigned to the no motor complaint group. Clinical characteristics and neuroimaging markers were compared between groups using student t-test for continuous variables and Chi-square test for dichotomous variables. General linear models were generated to compare each motor function parameters between groups. Model 1 adjusted for age and sex. Model 2 adjusted for age, sex and cerebrovascular risk factors.

To investigate whether age and sex were risk factors of motor complaints, we generated a binary logistic regression model including age and sex as predictor variables and motor complaints as dependent variable. To determine the association between motor complaints and cerebrovascular risk factors, binary logistic regression models were built. Model 1 adjusted for age and sex. Model 2 included age, sex, hypertension, diabetes, hyperlipidemia and smoking. 
To determine whether neuroimaging markers of CSVD were risk factors of motor complaints, we generated two kinds of binary logistic regression models. Model 1 adjusted only age and sex. Model 2 adjusted for Mini Mental State Examination (MMSE) scores and cerebrovascular risk factors such as hypertension, diabetes, hyperlipidemia and smoking, in addition to factors on Model 1.

\section{Results}

A total of 1586 participants joined the Shunyi Study. Among them, 329 participants refused or had contradictions against magnetic resonance imaging (MRI) scanning, leaving 1257 (79\%) participants with brain MRI scans. We step-wisely excluded 47 participants because of a history of ischemic/hemorrhagic stroke, 107 participants because not accomplishing Tanner's standard questionnaire, and 15 participants because having muscle strength less than four of any limb (Figure 1.). Among the remaining 1088 participants, no one had a medical history of Parkinson's disease. Individuals less than 50 years old were excluded since motor complaints were rarely seen in this age group. A total of 854 participants (male, 38.6\%) were finally analyzed. The average age was 60.7 years old. Demographic, clinical and imaging markers are shown in Table 1. Based on our questionnaire, 133 (15.6\%) individuals had at least one motor complaint. The most reported complaint on the questionnaire was having difficulty fastening buttons (6.1\%).

Table 2. Shows motor function parameters between the two groups. After adjusting for age and sex, finger-tapping time was positively associated with motor complaints (standardized $\beta=0.50, S E=0.19, p$ $=0.009$ ), and time for $3 \mathrm{~m}$-walking was positively associated with motor complaints (standardized $\beta=$ $0.26, \mathrm{SE}=0.11, \mathrm{p}=0.014)$. The association remained after additional adjustment for cerebrovascular risk factors, i.e. hypertension, diabetes, hyperlipidemia, and smoking. Finger-tapping time was positively associated with motor complaints (standardized $\beta=0.41, \mathrm{SE}=0.19, \mathrm{p}=0.037$ ), and time for $3 \mathrm{~m}$-walking was positively associated with motor complaints (standardized $\beta=0.21, S E=0.10, p=0.034$ ). we did not find association between motor complaints and stand-up time or pronation time.

Association between motor complaints and cerebrovascular risk factors are shown in Table 3. Hypertension was associated with motor complaints (odds ratio, 1.79; 95\% $\mathrm{Cl},[1.2,2.67] ; \mathrm{p}=0.005$ ) after adjusting for age and sex. The association still existed (odds ratio, 1.82; 95\% $\mathrm{Cl},[1.21,2.73], \mathrm{p}=0.004$ ) after additional adjustment for other cerebrovascular risk factors, i.e. diabetes, hyperlipidemia and smoking. There was a tendency of association between age and motor complaints (odds ratio, 1.27; $95 \%$ $\mathrm{Cl},[1.00,1.61] ; \mathrm{p}=0.052)$ after adjusting for sex. No association was found between age and motor complaints after adjusting for sex and other cerebrovascular risk factors (hypertension, diabetes, hyperlipidemia, smoking). No association was found between motor complaints and sex, diabetes, hyperlipidemia, or smoking.

The relationship between motor complaints and CSVD markers are shown in Table 4. No association was found between CSVD markers and motor complaints in both univariate analysis, multivariate analysis 
adjusting for age and sex, or multivariate analysis adjusting for age, sex, MMSE and cerebrovascular risk factors.

\section{Discussion}

Our study found that in a group of well-functioning, independently living, elderly adults in a Chinese rural area, individuals with Parkinsonism-related motor complaints had slower gait speed and slower fingertapping. Parkinsonism-related motor complaints were associated with hypertension. No association was found between Parkinsonism-related motor complaints and CSVD markers or cerebrovascular risk factors (hypertension, diabetes, hyperlipidemia, and smoking).

When screening for incidence of Parkinsonism in a community population, questionnaires had higher sensitivity and lower specificity, and trained medical staff had lower sensitivity and higher specificity[12]. Tanner's standard questionnaire is the most widely tested and used questionnaire in screening Parkinsonism, with a very high sensitivity $[12,13]$. In our community sample, symptoms associated with bradykinesia and rigidity was found to be two of the most common motor complaints. When measured objectively, slower finger-tapping, and slower $3 \mathrm{~m}$-walking speed, signs of bradykinesia, were found in the motor complaint group. These findings suggest that individuals with subjective motor complaints tend to have worse objectively measured motor performance.

Among all cerebrovascular risk factors, hypertension was found to be associated with motor complaints. In previous studies, Pinter et al. found that age, gender and hypertension were the major predicting factors of gait velocity[5], and Rosano et al. found that hypertension speeds up the gait velocity decline in well-functioning elderly populations[10]. These findings were in agreement with our results. This result could not be explained by structural CSVD brain changes since no association was found between CSVD markers and motor complaints. Subclinical damages of the peripheral vessels, nerves, bones, and muscles due to hypertension might be the cause of motor complaints[10,17]. Although age was proven to be associated with signs of Parkinsonism[8] and showed a tendency of association with Parkinsonism-related motor complaints when only adjusting for sex, no statistically significant association was found after additional adjustment of cerebrovascular risk factors. The reason might be that elderly people often attribute the decline of their motor performance to aging rather than report them as motor complaints.

No association was found between CSVD markers and motor complaints in univariate analysis and multivariate analysis adjusting only for age and sex or adjusting for age, sex, MMSE and cerebrovascular risk factors (hypertension, diabetes, hyperlipidemia, and smoking). Hence, the questionnaire, which aimed for Parkinsonism, is not suitable to screen CSVD in community-dwelling population.

A few studies have focused on the CSVD markers and motor disorders. In the LADIS study, WMH was associated with worse gait and balance cross-sectionally[18], and a more severe baseline WMH was associated with worse gait and balance in the follow up[19]. In the RUN-DMC study, WMH, lacunes, and CMBs were all found to be independently associated with gait disorders[4, 10]. WMH and lacunes were 
found to be associated with mild parkinsonism syndrome[3]. In a study conducted by Reiz et al., large $\mathrm{WMH}$ and lacunes were related with increased risk of resting tremor, and brain atrophy was related with increased risk of rigidity and bradykinesia[1]. In another study conducted by Hatate et al., people with at least one cerebrovascular risk factors were included and WMH, lacunes, CMB, and total CSVD burden scores were all associated with higher incidence of mild Parkinsonism syndrome[2]. However, unlike in the studies above[1, 2, 20,21], since we partly included people without any cerebrovascular risk factors and excluded people who with a history of ischemic or hemorrhagic stroke, the prevalence of CSVD and cerebrovascular risk factors were lower in our study population. As observed among the elderly population, subjective motor complaints do not increase proportionately with objectively worsened motor performance. Thus, the association between CSVD markers and motor complaints may be weakened.

Motor disorders, especially declining gait speed, were proven to impair the ability for independently living and increase the risk of institutionalization and mortality[22-24]. Although motor complaints may not coincide with objectively measured motor deficits, they directly limit daily activities, generate sedentary lifestyles, and promote cerebrovascular risk factors. Therefore, the association between motor complaints and cerebrovascular risk factors reinforces itself in a vicious circle. Screening for those who are at risk and controlling the risk factors would prevent them from falling into that vicious circle, improve their quality of living and reduce the cost of health care.

The strength of our study is the inclusion of a large sample of community-dwelling population. In addition, it is the first study focusing on association of Parkinsonism- related motor complaints with cerebrovascular risk factors and CSVD.

There are three limitations to be considered. First, the questionnaire we used was designed to screen Parkinsonism-related motor disorders. It could not evaluate motor deficits that generates from non-CNS diseases such as arthritis, myopathy, or peripheral neuropathy. Second, we excluded individuals who had a history of ischemic or hemorrhagic stroke. Considering the high prevalence of cerebrovascular disease among the elderly, our study may underestimate the prevalence and severity of both CSVD and motor complaints, which kept us from generalizing our results to the total elderly population. Third, we did not look further into the time course, degree, and control of hypertension. We hope that future studies would explore this further.

\section{Conclusion}

We found that in a group of well-functioning, independently living elderly population from a Chinese rural area, Parkinsonism-related motor complaints were associated with hypertension. No association was found between motor complaint and age or CSVD markers. Tanner's standard questionnaire for parkinsonism is not suitable for screening of CSVD in a community-dwelling population.

\section{Declarations}


Ethics approval and consent to participate

The Shunyi study was approved by the Ethical Committee at Peking Union Medical College Hospital (reference number: B-160).

\section{Consent for publication}

Written informed consent were obtained from all participants during inclusion process.

\section{Availability of data and materials}

The datasets used and analyzed during the current study are available from corresponding author on reasonable request.

\section{Competing interests}

The authors declare that they have no competing interests.

\section{Funding}

This study was funded by the National Natural Science Foundation of China (grant number: 81173663), and the National Key Technology R\&D Program in the $12^{\text {th }}$ Five-year Plan of China (grant numbers: 2015AA020506, 2016YFC0901004, and 2016YFC1300500-505).

\section{Authors' Contributions}

YG drafted the manuscript and conducted the statistical analysis. CJ collected the questionnaires. FH, JZ and FZ managed the database. LZ, JN, MY, ZJ, SZ, LC, and YZ contributed to the conception and design of the study and interpretation of the data. All authors provided final approval for the version of the manuscript submitted for publication and were accountable for the work. YZ was the principal investigator of the study; she was responsible for the study conception and interpretation of data and had final responsibility for the decision to submit for publication.

Acknowledgements

Not applicable.

\section{References}

1.Reitz C, Trenkwalder C, Kretzschmar K, Roesler A, A VE, Berger K: Relation of cerebral small-vessel disease and brain atrophy to mild Parkinsonism in the elderly. Mov Disord 2006, 21(11):1914-1919.

2. Hatate J, Miwa K, Matsumoto M, Sasaki T, Yagita Y, Sakaguchi M, Kitagawa K, Mochizuki H: Association between cerebral small vessel diseases and mild parkinsonian signs in the elderly with vascular risk factors. Parkinsonism \& Related Disorders 2016, 26:29-34. 
3.de Laat KF, van Norden AG, Gons RA, van Uden IW, Zwiers MP, Bloem BR, van Dijk EJ, de Leeuw FE: Cerebral white matter lesions and lacunar infarcts contribute to the presence of mild parkinsonian signs. Stroke 2012, 43(10):2574-2579.

4.de Laat KF, van Norden AGW, Gons RAR, van Oudheusden LJB, van Uden IWM, Bloem BR, Zwiers MP, de Leeuw F-E: Gait in Elderly With Cerebral Small Vessel Disease. Stroke 2010, 41(8):1652-1658.

5.Pinter D, Ritchie SJ, Doubal F, Gattringer T, Morris Z, Bastin ME, del C. Valdés Hernández M, Royle NA, Corley J, Muñoz Maniega S et al: Impact of small vessel disease in the brain on gait and balance. Scientific Reports 2017, 7(1).

6.Kim YJ, Kwon HK, Lee JM, Cho H, Kim HJ, Park HK, Jung NY, San Lee J, Lee J, Jang YK et al: Gray and white matter changes linking cerebral small vessel disease to gait disturbances. Neurology 2016, 86(13):1199-1207.

7.de Laat KF, van den Berg HAC, van Norden AGW, Gons RAR, Olde Rikkert MGM, de Leeuw F-E: Microbleeds Are Independently Related to Gait Disturbances in Elderly Individuals With Cerebral Small Vessel Disease. Stroke 2011, 42(2):494-497.

8.Bennett DA, Beckett LA, Murray AM, Shannon KM, Goetz CG, Pilgrim DM, Evans DA: Prevalence of parkinsonian signs and associated mortality in a community population of older people. $\mathrm{N}$ Engl J Med 1996, 334(2):71-76.

9.Su N, Zhai F-F, Zhou L-X, Ni J, Yao M, Li M-L, Jin Z-Y, Gong G-L, Zhang S-Y, Cui L-Y et al: Cerebral Small Vessel Disease Burden Is Associated with Motor Performance of Lower and Upper Extremities in Community-Dwelling Populations. Frontiers in Aging Neuroscience 2017, 9.

10.Rosano C, Longstreth WT, Boudreau R, Taylor CA, Du Y, Kuller LH, Newman AB: High Blood Pressure Accelerates Gait Slowing in Well-Functioning Older Adults over 18-Years of Follow-Up. Journal of the American Geriatrics Society 2011, 59(3):390-397.

11.Tanner CM, Gilley DW, Goetz CG: A Brief Screening Questionnaire for Parkinsonism. Annals of Neurology 1990, 28(2):267-268.

12.Höglinger GU, Rissling I, Metz A, Ries V, Heinermann A, Prinz H, Spieker S, Deuschl G, Baum E, Oertel WH: Enhancing recognition of early Parkinsonism in the community. Movement Disorders 2004, 19(5):505-512.

13.Dahodwala N, Siderowf A, Baumgarten M, Abrams A, Karlawish J: Screening questionnaires for parkinsonism: A systematic review. Parkinsonism \& Related Disorders 2012, 18(3):216-224.

14. Wardlaw JM, Smith EE, Biessels GJ, Cordonnier C, Fazekas F, Frayne R, Lindley RI, O'Brien JT, Barkhof $\mathrm{F}$, Benavente $\mathrm{OR}$ et al: Neuroimaging standards for research into small vessel disease and its contribution to ageing and neurodegeneration. The Lancet Neurology 2013, 12(8):822-838. 
15.Fazekas F, Chawluk JB, Alavi A, Hurtig HI, Zimmerman RA: MR signal abnormalities at $1.5 \mathrm{~T}$ in Alzheimer's dementia and normal aging. AJR Am J Roentgenol 1987, 149(2):351-356.

16.Zhu Y-C, Tzourio C, Soumaré Ac, Mazoyer B, Dufouil C, Chabriat H: Severity of Dilated Virchow-Robin Spaces Is Associated With Age, Blood Pressure, and MRI Markers of Small Vessel Disease. Stroke 2010, 41(11):2483-2490.

17.Rubino FA: Gait disorders. The neurologist 2002, 8(4):254-262.

18.Baezner H, Blahak C, Poggesi A, Pantoni L, Inzitari D, Chabriat H, Erkinjuntti T, Fazekas F, Ferro JM, Langhorne $\mathrm{P}$ et al: Association of gait and balance disorders with age-related white matter changes: The LADIS Study. Neurology 2008, 70(12):935-942.

19.Kreisel SH, Blahak C, Bazner H, Inzitari D, Pantoni L, Poggesi A, Chabriat H, Erkinjuntti T, Fazekas F, Ferro JM et al: Deterioration of gait and balance over time: the effects of age-related white matter change -the LADIS study. Cerebrovasc Dis 2013, 35(6):544-553.

20.Pantoni L, Basile AM, Pracucci G, Asplund K, Bogousslavsky J, Chabriat H, Erkinjuntti T, Fazekas F, Ferro JM, Hennerici M et al: Impact of age-related cerebral white matter changes on the transition to disability-the LADIS study: rationale, design and methodology. Neuroepidemiology 2005, 24(1-2):5162 .

21.van Norden AG, de Laat KF, Gons RA, van Uden IW, van Dijk EJ, van Oudheusden LJ, Esselink RA, Bloem BR, van Engelen BG, Zwarts MJ et al: Causes and consequences of cerebral small vessel disease. The RUN DMC study: a prospective cohort study. Study rationale and protocol. BMC Neuro/ 2011, 11:29.

22.Studenski S: Gait Speed and Survival in Older Adults. Jama 2011, 305(1).

23.van der Holst HM, van Uden IWM, Tuladhar AM, de Laat KF, van Norden AGW, Norris DG, van Dijk EJ, Rutten-Jacobs LC, de Leeuw F-E: Factors Associated With 8-Year Mortality in Older Patients With Cerebral Small Vessel Disease. JAMA Neurology 2016, 73(4).

24.Verghese J, LeValley A, Hall CB, Katz MJ, Ambrose AF, Lipton RB: Epidemiology of Gait Disorders in Community-Residing Older Adults. Journal of the American Geriatrics Society 2006, 54(2):255-261.

\section{Tables}

Table 1. Baseline characteristics of the study population 


\begin{tabular}{|c|c|c|c|c|}
\hline $\begin{array}{l}\text { Clinical } \\
\text { Characteristics }\end{array}$ & Total & $\begin{array}{c}\text { Motor } \\
\text { complaints }\end{array}$ & $\begin{array}{l}\text { No motor } \\
\text { complaints }\end{array}$ & $\begin{array}{c}\mathrm{P} \\
\text { value }\end{array}$ \\
\hline Age (year) & $60.7(7.6)$ & $61.8(7.6)$ & $60.5(7.6)$ & 0.056 \\
\hline Sex (male\%) & $330(38.6 \%)$ & $48(36.1 \%)$ & $282(39.1 \%)$ & 0.561 \\
\hline Hypertension & $479(56.1 \%)$ & $91(68.4 \%)$ & $388(53.8 \%)$ & $0.002^{*}$ \\
\hline Diabetes & 157 (18.4\%) & $22(16.5 \%)$ & 135 (18.7\%) & 0.627 \\
\hline Hyperlipidemia & $453(53.0 \%)$ & $72(54.1 \%)$ & $381(52.8 \%)$ & 0.850 \\
\hline Smoking & $200(23.8 \%)$ & $26(19.6 \%)$ & $174(24.5 \%)$ & 0.224 \\
\hline MMSE & $25.7(3.9)$ & $24.8(4.4)$ & $25.8(3.8)$ & $0.007^{*}$ \\
\hline \multicolumn{5}{|c|}{ Neuroimaging markers } \\
\hline $\begin{array}{l}\text { Total WMH volume } \\
\square \mathrm{ml}\end{array}$ & $3.17(5.47)$ & $3.73(7.35)$ & $3.08(5.09)$ & 0.169 \\
\hline PVWMH & & & & 0.742 \\
\hline Mild & $646(75.6 \%)$ & $99(74.4 \%)$ & $547(75.9 \%)$ & \\
\hline Severe & $208(24.4 \%)$ & $34(25.6 \%)$ & $174(24.1 \%)$ & \\
\hline DWMH & & & & 1.000 \\
\hline Mild & $732(85.7 \%)$ & $114(85.7 \%)$ & $618(85.7 \%)$ & \\
\hline Severe & $122(14.3 \%)$ & $19(14.3 \%)$ & $103(14.3 \%)$ & \\
\hline PVS-BG & & & & 0.899 \\
\hline Degree 1 or 2 & $712(83.8 \%)$ & $111(83.5 \%)$ & 601 (83.8\%) & \\
\hline Degree 3 or 4 & $138(16.2 \%)$ & $22(16.5 \%)$ & $116(16.2 \%)$ & \\
\hline PVS-WM & & & & 0.615 \\
\hline Degree 1 or 2 & $708(83.3 \%)$ & $113(85.0 \%)$ & 595 (83.0\%) & \\
\hline Degree 3 or 4 & $142(16.7 \%)$ & $20(15.0 \%)$ & $122(17.0 \%)$ & \\
\hline Presence of Lacunes & $160(18.7 \%)$ & $27(20.3 \%)$ & $133(18.5 \%)$ & 0.629 \\
\hline Presence of CMBs & $108(12.7 \%)$ & $17(12.8 \%)$ & 91 (12.6\%) & 1.000 \\
\hline Total Brain Volume & $\begin{array}{l}1402.4 \\
(122.5)\end{array}$ & $1404.9(121.8)$ & $1388.0(125.8)$ & $0.010^{*}$ \\
\hline $\mathrm{BPF}$ & $0.76(0.03)$ & $0.75(0.03)$ & $0.76(0.03)$ & 0.281 \\
\hline \multicolumn{5}{|c|}{ Motor function parameters } \\
\hline 3m-walking (s) & $3.7(1.1)$ & $4.0(1.6)$ & $3.6(1.0)$ & 0.152 \\
\hline Stand-up time (s) & $9.2(2.3)$ & $9.6(2.9)$ & $9.2(2.2)$ & 0.246 \\
\hline Pronation time (s) & $7.7(1.7)$ & $7.8(1.8)$ & $7.7(1.7)$ & 0.829 \\
\hline Finger tapping (s) & $5.7(1.9)$ & $6.2(1.9)$ & $5.6(1.9)$ & $0.002^{*}$ \\
\hline
\end{tabular}

Abbreviations: MMSE: mini mental state examination; WMH: white matter hyperintensity; PVWMH: periventricular white matter hyperintensity; DWMH: deep white matter 
hyperintensity; PVS-BG: perivascular spaces in basal ganglia; PVS-WM: perivascular spaces in white matter; CMB: cerebral microbleed; BPF: brain parenchymal fraction.

$* \mathrm{p}<0.05$

Table 2. Motor function parameters of people with or without motor complaints

\begin{tabular}{lccccc}
\hline \multirow{2}{*}{ Motor function } & \multicolumn{2}{c}{ Model 1} & & \multicolumn{2}{c}{ Model 2} \\
\cline { 2 - 3 } \cline { 5 - 6 } \cline { 5 - 6 } & $\beta(\mathrm{SE})$ & P value & & $\beta(\mathrm{SE})$ & P value \\
\hline 3m-walking & $0.26(0.11)$ & $0.014^{*}$ & & $0.21(0.10)$ & $0.034^{*}$ \\
Stand-up time & $0.28(0.24)$ & 0.232 & & $0.20(0.24)$ & 0.401 \\
Pronation time & $0.07(0.17)$ & 0.684 & & $-0.07(0.17)$ & 0.683 \\
Finger tapping & $0.50(0.19)$ & $0.009^{*}$ & & $0.41(0.19)$ & $0.037^{*}$ \\
\hline
\end{tabular}

General linear model was used.

$\beta$ : standardized $\beta$ coefficient; SE: standard error.

Model 1: adjusting for age and sex.

Model 2: additional adjustment for MMSE and vascular risk factors (hypertension, diabetes, hyperlipidemia, smoking) on Model 1.

${ }^{*} \mathrm{p}<0.05$.

Table 3. Association between vascular risk factors and motor complaints 


\begin{tabular}{lllllll}
\hline \multirow{2}{*}{ Vascular risk factors } & \multicolumn{3}{c}{ Model 1} & & \multicolumn{2}{c}{ Model 2 } \\
\cline { 2 - 3 } \cline { 6 - 7 } & OR $(95 \%$ CI $)$ & P value & & OR $(95 \%$ CI $)$ & P value \\
\hline Age & $1.27(1.00,1.61)^{\mathrm{a}}$ & 0.052 & & $1.19(0.93,1.53)^{\mathrm{d}}$ & 0.163 \\
Sex (male) & $0.86(0.59,1.27)^{\mathrm{b}}$ & 0.447 & & $0.93(0.57,1.54)^{\mathrm{d}}$ & 0.788 \\
Hypertension & $1.79(1.20,2.67)^{\mathrm{C}}$ & $0.005^{*}$ & & $1.82(1.21,2.73)^{\mathrm{d}}$ & $0.004^{*}$ \\
Diabetes & $0.81(0.49,1.33)^{\mathrm{C}}$ & 0.405 & & $0.79(0.48,1.31)^{\mathrm{d}}$ & 0.366 \\
Hyperlipidemia & $1.00(0.68,1.46)^{\mathrm{c}}$ & 0.986 & & $0.92(0.62,1.36)^{\mathrm{d}}$ & 0.666 \\
Smoking & $0.74(0.42,1.32)^{\mathrm{C}}$ & 0.3122 & & $0.78(0.43,1.39)^{\mathrm{d}}$ & 0.396 \\
\hline
\end{tabular}

a: adjusting for sex.

b: adjusting for age.

c: adjusting for age and sex.

d: including age, sex, cerebrovascular risk factors (hypertension, diabetes, hyperlipidemia and smoking).

OR: odds ratio.

$* \mathrm{p}<0.05$.

Table 4. Association between CSVD markers and motor complaints

\begin{tabular}{llllll}
\hline CSVD markers & \multicolumn{3}{c}{ Model 1} & & \multicolumn{2}{c}{ Model 2} \\
\cline { 2 - 3 } \cline { 5 - 6 } & OR $(95 \%$ CI $)$ & P value & & OR $(95 \%$ CI $)$ & P value \\
\hline Total WMH & $0.84(0.53,1.32)$ & 0.442 & & $0.78(0.48,1.25)$ & 0.298 \\
DWMH & $0.87(0.50,1.51)$ & 0.630 & & $0.79(0.45,1.40)$ & 0.418 \\
PVWMH & $0.91(0.57,1.45)$ & 0.691 & & $0.83(0.51,1.34)$ & 0.434 \\
Lacunes & $1.02(0.62,1.67)$ & 0.943 & & $0.93(0.55,1.56)$ & 0.778 \\
CMBs & $0.93(0.53,1.65)$ & 0.807 & & $0.83(0.46,1.51)$ & 0.551 \\
PVS-BG & $0.96(0.58,1.60)$ & 0.884 & & $1.03(0.62,1.72)$ & 0.911 \\
PVS-WM & $0.85(0.51,1.43)$ & 0.539 & & $0.84(0.50,1.43)$ & 0.52 \\
BPF & $0.98(0.90,1.07)$ & 0.727 & & $0.96(0.87,1.05)$ & 0.321 \\
\hline
\end{tabular}

Model 1: adjustment for age and sex. 
Model 2: additional adjustment for MMSE and cerebrovascular risk factors (hypertension, diabetes, hyperlipidemia, and smoking).

Abbreviations: CSVD, cerebral small vessel disease; DWMH, deep white matter hyperintensity; $\mathrm{WMH}$, white matter hyperintensity; PVWMH, periventricular white matter hyperintensity; CMB, cerebral microbleed; PVS-BG, perivascular spaces in the basal ganglia; PVS-WM, perivascular spaces in white matter; BPF, brain parenchymal fraction.

OR: odds ratio.

${ }^{*} \mathrm{p}<0.05$.

\section{Figures}


Total sample 1586

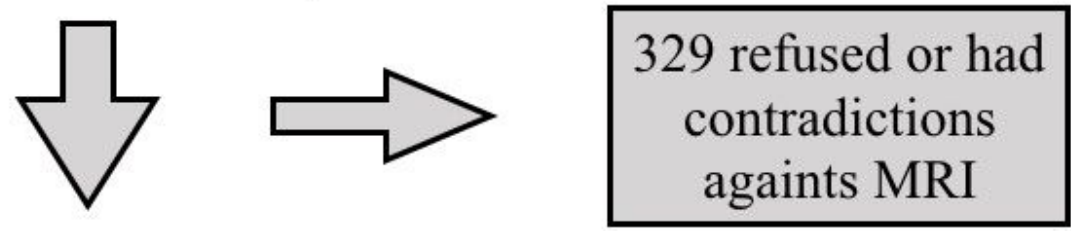

1257 completed MRI
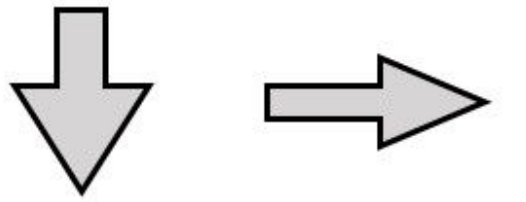

47 had a history of stroke

1210 had taken Tanner's standard questionnaire

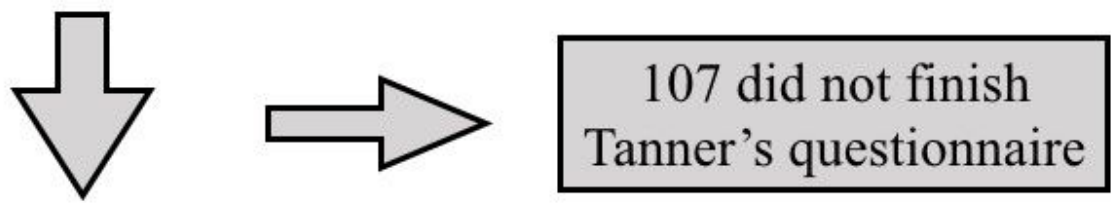

1103 individuals
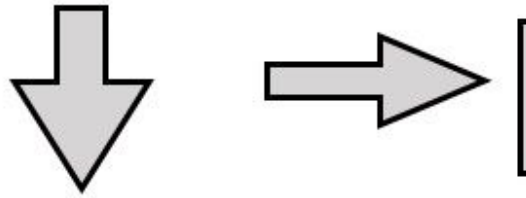

15 had muscle strength less than 4 of any limb

1088 individuals
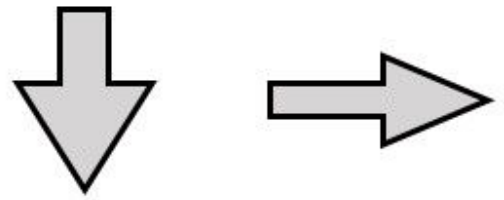

234 younger than 50 years old

854 individuals

Figure 1

Study profile. 